\title{
Comparative analyses of two Geraniaceae transcriptomes using next-generation sequencing
}

\author{
Jin Zhang ${ }^{1}$, Tracey A Ruhlman ${ }^{1}$, Jeffrey P Mower ${ }^{2}$ and Robert K Jansen ${ }^{1,3^{*}}$
}

\begin{abstract}
Background: Organelle genomes of Geraniaceae exhibit several unusual evolutionary phenomena compared to other angiosperm families including accelerated nucleotide substitution rates, widespread gene loss, reduced RNA editing, and extensive genomic rearrangements. Since most organelle-encoded proteins function in multi-subunit complexes that also contain nuclear-encoded proteins, it is likely that the atypical organellar phenomena affect the evolution of nuclear genes encoding organellar proteins. To begin to unravel the complex co-evolutionary interplay between organellar and nuclear genomes in this family, we sequenced nuclear transcriptomes of two species, Geranium maderense and Pelargonium x hortorum.

Results: Normalized cDNA libraries of G. maderense and $P . x$ hortorum were used for transcriptome sequencing. Five assemblers (MIRA, Newbler, SOAPdenovo, SOAPdenovo-trans [SOAPtrans], Trinity) and two next-generation technologies (454 and Illumina) were compared to determine the optimal transcriptome sequencing approach. Trinity provided the highest quality assembly of Illumina data with the deepest transcriptome coverage. An analysis to determine the amount of sequencing needed for de novo assembly revealed diminishing returns of coverage and quality with data sets larger than sixty million Illumina paired end reads for both species. The G. maderense and P. $\times$ hortorum transcriptomes contained fewer transcripts encoding the PLS subclass of PPR proteins relative to other angiosperms, consistent with reduced mitochondrial RNA editing activity in Geraniaceae. In addition, transcripts for all six plastid targeted sigma factors were identified in both transcriptomes, suggesting that one of the highly divergent rpoA-like ORFs in the $P . \times$ hortorum plastid genome is functional.

Conclusions: The findings support the use of the Illumina platform and assemblers optimized for transcriptome assembly, such as Trinity or SOAPtrans, to generate high-quality de novo transcriptomes with broad coverage. In addition, results indicated no major improvements in breadth of coverage with data sets larger than six billion nucleotides or when sampling RNA from four tissue types rather than from a single tissue. Finally, this work demonstrates the power of cross-compartmental genomic analyses to deepen our understanding of the correlated evolution of the nuclear, plastid, and mitochondrial genomes in plants.
\end{abstract}

\section{Background}

Four remarkable evolutionary phenomena are associated with organellar genomes of Geraniaceae. First, mitochondrial genomes show multiple, major shifts in rates of synonymous substitutions, especially in the genus Pelargonium $[1,2]$. Rate fluctuations of such magnitude have been documented in only two other plant lineages, Plantago

\footnotetext{
* Correspondence: jansen@austin.utexas.edu

'Department of Integrative Biology and Institute of Cellular and Molecular Biology, The University of Texas at Austin, 205 W. 24th St. Stop C0930, Austin, TX 78712, USA

${ }^{3}$ Genomics and Biotechnology Section, Department of Biological Sciences, Faculty of Science, King Abdulaziz University, Jeddah 21589, Saudi Arabia Full list of author information is available at the end of the article
}

[3] and Silene [4-6]. Second, mitochondrial genomes have experienced extensive loss of genes and sites of RNA editing. At least 12 putative gene losses have been documented in Erodium [7], and mitochondrial genes sequenced from Pelargonium x hortorum had a drastic reduction in predicted or verified RNA editing sites compared to all other angiosperms examined [1]. Third, genome-wide comparisons of nucleotide substitutions in plastid DNA indicated rapid rate acceleration in genes encoding ribosomal proteins, RNA polymerase, and ATP synthase subunits in some lineages. In the case of RNA polymerase genes there was evidence for positive selection $[8,9]$. Fourth, plastid genomes of Geraniaceae

\section{Biomed Central}

(c) 2013 Zhang et al.; licensee BioMed Central Ltd. This is an open access article distributed under the terms of the Creative Commons Attribution License (http://creativecommons.org/licenses/by/2.0), which permits unrestricted use, distribution, and reproduction in any medium, provided the original work is properly cited. 
are the most highly rearranged of any photosynthetic land plants examined [10-13]. Multiple and extreme contractions and expansions of the inverted repeat (IR) have resulted in genomes with both the largest IR (74,571 bp, [11]) as well as the complete loss of this feature $[12,13]$. Considerable accumulation of dispersed repeats associated with changes in gene order has been documented along with disruption of highly conserved operons and repeated losses and duplications of genes [12]. In P. x hortorum plastids, these genomic changes have generated several fragmented and highly divergent $r p o A$-like ORFs of questionable functionality [8,10-12], despite the fact that rpoA encodes an essential component of the plastid-encoded RNA polymerase (PEP).

Because nuclear genes supply both organelles with the majority of their proteins, it is likely that the extensive organellar genomic upheaval in Geraniaceae will also influence the evolution of organelle-targeted genes in the nuclear genome. For example, given the drastic reduction of RNA editing in Geraniaceae mitochondrial transcripts, it is reasonable to expect a correlated reduction of nucleus-encoded pentatricopeptide repeat (PPR) proteins, many of which are critical for organellar RNA editing [14-17]. The uncertain status of the P. x hortorum plastid-encoded rpoA gene is also likely to have nuclear consequences. If this plastid gene is not functional, then a functional copy might have been relocated to the nuclear genome, which has only occurred once in the evolution of land plants in mosses $[18,19]$. Alternatively, it is possible that PEP has become nonfunctional in $P . \mathrm{x}$ hortorum, as observed in the holoparasite Phelipanche aegyptiaca [20]. In $P$. aegyptiaca, loss of all plastid-encoded PEP components (rpoA, rрoB, rpoC1 and rрoC2) resulted in the parallel loss of the requisite nucleus-encoded components (sigma factors) that assemble with the plastid encoded proteins to form the core of the PEP holoenzyme [20]. In contrast, if the highly divergent plastid rpoA gene is still functional in $P . \mathrm{x}$ hortorum, then the typical set of sigma factors should be present in the nuclear genome.

One prerequisite to begin to address the effects of organellar genomic upheaval on the nuclear genome in Geraniaceae, is availability of nuclear sequence information. Transcriptome sequencing provides a tractable proxy for nuclear gene space. The use of next-generation sequencing (NGS) for transcriptome sequencing is widespread because volumes of data can be generated rapidly at a low cost relative to traditional Sanger sequencing [21]. The assembly of reads into contigs may be executed using a de novo or a reference-based approach [22]. In studies of non-model organisms, de novo assembly is more commonly used due to the absence of a closely related reference $[23,24]$. A survey of recent transcriptome studies in comparative biology demonstrates that most sequencing projects are focusing on non-model organisms where little or no genomic data is available [22,25-31]. The lack of a reference genome makes the reconstruction and evaluation of the transcriptome assembly challenging. Several issues must be addressed when performing transcriptome sequencing of non-model organisms, including which NGS platform should be employed, how much sequence data is needed to provide a comprehensive transcriptome, which assembler should be utilized, and what tissues should be sampled.

This paper provides a comprehensive comparison of the transcriptomes of two non-model plant species, Pelargonium $\mathrm{x}$ hortorum and Geranium maderense, from the two largest genera of Geraniaceae. There were three primary goals for the initial comparative transcriptome analysis in Geraniaceae: (1) What are the best sequencing platforms and assembly methods for generating a high-quality transcriptome that broadly covers gene space in the absence of a reference genome? (2) Does sequencing from multiple tissue types improve the breadth of transcriptome coverage? (3) Are there any losses of PPR proteins involved in RNA editing and sigma factors associated with PEP in Geraniaceae?

\section{Results}

Ribosomal RNA content and Illumina library complexity

To assess the efficiency of ribosomal RNA (rRNA) depletion in Geraniaceae transcriptome libraries rRNA contigs were identified using rRNA from Arabidopsis thaliana as a reference. All Illumina reads $(146,690,142$ reads for Geranium maderense and 148,749,374 reads for Pelargonium $\mathrm{x}$ hortorum) were mapped to rRNA contigs as described in methods, and $0.7 \%$ and $2 \%$ of the reads of G. maderense and P. x hortorum were identified as rRNA reads, respectively. Library complexity was analyzed using Picard [32] and rRNA reads were eliminated prior to the analysis. The percentages of unique start sites were $42.7 \%$ and $46.1 \%$ for G. maderense and P. x hortorum, respectively. The values for rRNA content and library complexity were comparable to other transcriptome analyses using similar approaches [33,34].

\section{Assessment of sequencing platforms and assemblers for transcriptome assembly}

To determine the optimal sequencing and assembly strategy, the efficacy of five different assemblers was examined using two initial data sets generated by Roche/ 454 FLX and Illumina Hiseq 2000 platforms for P. x hortorum. The Illumina run produced approximately 40 times more sequence data than the 454 run, even though the cost of the 454 data was at least four times more than the Illumina data (Table 1). A comparison of basic assembly statistics (Table 2 ) showed that the Trinity assembler outperformed all other platform/software combinations in terms of number of contigs, number of assembled nucleotides, mean and maximum contig length, and 
Table 1 The Pelargonium $\mathrm{x}$ hortorum transcriptome dataset read statistics

\begin{tabular}{lllll}
\hline Technology & Number of trimmed reads & Number of trimmed bases & Max read length & Min read length \\
\hline $\mathbf{4 5 4}$ & 472,268 & $119,394,317$ & 828 & 50 \\
Illumina & $46,475,742$ & $4,674,574,200$ & 100 & 100 \\
\hline
\end{tabular}

N50. More generally, the Illumina assemblers consistently outperformed the 454 assemblers, although the MIRA and Newbler 454 assemblers produced longer maximal contigs than SOAPdenovo and SOAPdenovo-trans (SOAPtrans). To determine the amount of usable protein sequence information generated by each assembler, the assemblies were translated as described in methods and compared (Table 3). Again, the Illumina assemblers outperformed the 454 assemblers in all metrics, with the Trinity assembler providing the most amino acids with the longest mean and maximal sequences. The length distribution of assembled nucleotides and translated amino acids further confirms that Trinity outperformed SOAPdenovo and SOAPtrans, and all three Illumina assemblers outperformed the 454 assemblers (Figure 1).

Two important considerations in assembly analysis are the breadth of gene space coverage and the degree of coverage fragmentation. A good assembler should generate highquality assemblies that contain as many reference transcripts as possible, and each reference transcript should be covered as completely as possible with a single long contig rather than a combination of several short contigs. To assess assembly coverage and fragmentation, two published data bases were used, 357 ultra-conserved ortholog (UCO) coding sequence [35] from Arabidopsis and 959 single copy nuclear genes shared between Arabidopsis, Oryza, Populus, and Vitis [36]. Trinity and SOAPtrans outperformed all other assemblers in terms of the percentage of reference genes identified, completeness of coverage (i.e. fraction of reference gene coverage by one or more contigs), and contiguity of coverage (i.e. fraction of reference gene coverage by a single long contig), with Trinity performance slightly better than SOAPtrans at higher thresholds (Figures 2 and 3).

To examine whether the superior performance of Trinity and SOAPtrans was due to the much larger amount (40 times) of Illumina data than 454 data, the Illumina assemblers were re-anlayzed using a data set containing $1 / 40^{\text {th }}$ of the Illumina reads (Additional file 1 ). In terms of contiguity and completeness, the performance of
Trinity using the reduced Illumina data set remained superior to the 454 programs (Newbler, MIRA) that used the entire 454 data sets. In contrast, the performance of SOAPdenovo and SOAPtrans were noticeably worse with the reduced Illumina data set than with the full data set, producing results that were generally worse than the original 454 assemblies.

\section{Effect of sequencing depth on assembly coverage breadth and fragmentation}

To determine how much sequence data is needed to assemble a high-quality transcriptome with broad coverage, 146,690,142 reads for G. maderense and 148,749,374 reads for $P . \mathrm{x}$ hortorum were generated on the Illumina Hiseq 2000 platform assembled using Trinity with different increments of reads from $5 \%$ to $100 \%$ of the total. While the number of contigs assembled continued to increase with increasing numbers of reads (Figure 4A), the percentage of reference genes recovered and their contiguity and completeness plateaued at approximately $40 \%$ of the total reads (Figure 4B-D). Including the remaining $60 \%$ of the reads increased contiguity and completeness by only $1 \%$ to $2 \%$ (Figure $4 \mathrm{~B}-\mathrm{C}$ ). Although there were more translated contigs of G. maderense than P. x hortorum, the contiguity and completeness of both species were very similar.

Although increasing the number of reads beyond $10 \%$ contributed little to finding novel hits to the local Arabidopsis data base, increasing the amount of data did help extend the existing contigs and generate longer alignments to reference genes. To evaluate this, the contiguity of all contigs relative to the two published databases was calculated at different contiguity thresholds up to $100 \%$ (Figure 5). The inclusion of more reads generated assemblies with higher contiguity, especially when contiguity thresholds were greater than $50 \%$. To allow for the high level of sequence divergence between Geraniaceae and Arabidopsis, the number of contigs that had contiguity thresholds $\geq 80 \%$ was calculated. When $100 \%$ of the reads were used 4185 contigs and 4494 contigs were found in

Table 2 Basic assembly statistics for the Pelargonium $\mathbf{x}$ hortorum transcriptome

\begin{tabular}{llllll}
\hline & Newbler & MIRA & SOAPdenovo & Trinity & SOAPtrans \\
\hline Number of nonredundant contigs & 28,182 & 30,947 & 67,028 & 67,614 & 62,470 \\
Total bases & $12,972,883$ & $15,326,277$ & $39,088,184$ & $58,210,111$ & $33,057,051$ \\
Max contig length & 8,147 & 12,431 & 6,616 & 16,017 & 7,574 \\
Mean contig length & 460 & 495 & 583 & 860 & 529 \\
N50 & 478 & 525 & 782 & 1,319 & 678 \\
\hline
\end{tabular}


Table 3 Translated contig statistics for Pelargonium $\mathbf{x}$ hortorum

\begin{tabular}{llllll}
\hline & Newbler & MIRA & SOAPdenovo & Trinity & SOAPtrans \\
\hline Number of translated contigs & 18,525 & 19,279 & 42,907 & 39,742 & 44,379 \\
Total amino acids (AA) & $2,413,770$ & $2,575,430$ & $8,363,275$ & $11,058,408$ & $7,697,127$ \\
Max translated AA length & 902 & 1,086 & 1,902 & 2,618 & 2,520 \\
Mean translated AA length & 130 & 133 & 195 & 278 & 173 \\
N50 & 145 & 145 & 278 & 387 & 230 \\
\hline
\end{tabular}

G. maderense and P. x hortorum, respectively. Reducing the read input to $40 \%$ reduced contiguity values by $7 \%$ (4163/4494) in G. maderense and $11 \%(3731 / 4185)$ in P. x hortorum.

\section{Functional assessment of Geraniaceae nuclear transcriptomes}

The assemblies generated using $100 \%$ of the reads for both Geraniaceae species were used for functional annotation. Assemblies were first aligned against the NCBI nr database and the alignment results were used to generate the gene ontology (GO) terms. Of the 114,762 contigs in $P$. $\mathrm{x}$ hortorum, 56,283 (49\%) had blast hits; 42,506 (37\%) were annotated and 222,765 GO terms were retrieved (Table 4). Of the 119,217 contigs in G. maderense, 76,332 (64\%) had blast hits; 58,461 (49\%) were annotated (Table 4) and 311,108 GO terms were retrieved. The annotation files are shown in Additional file 2. The distribution of gene ontology annotations was examined using GO-slim (plant) ontology to compare the transcriptomes of $G$. maderense and P. x hortorum. Although the number of annotated contigs differed substantially between the two transcriptomes (Table 4), the proportion of annotated contigs in all categories with $>1 \%$ representation within
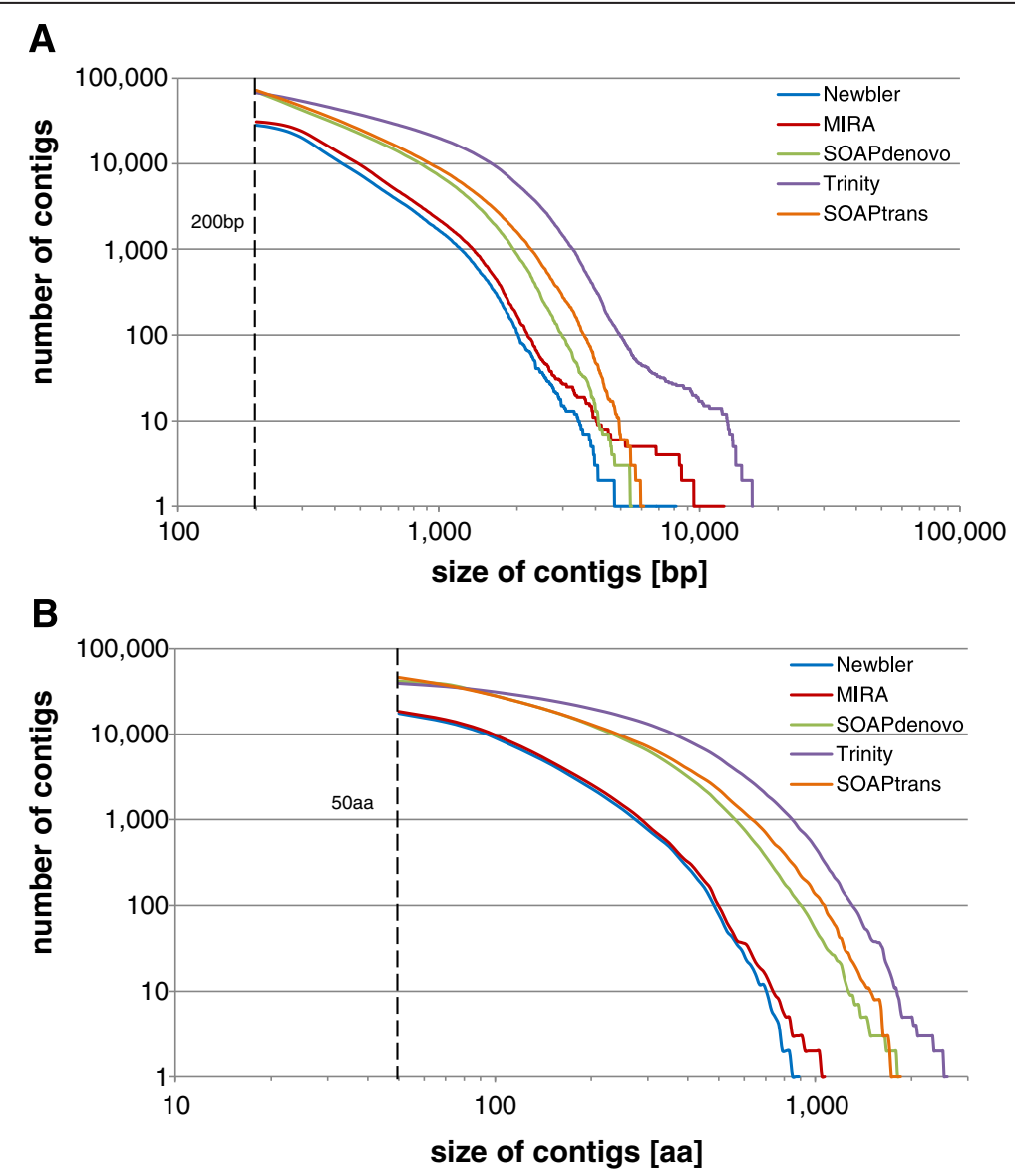

Figure 1 Contig length. (A) Contig length distribution. The vertical dashed line shows the arbitrary cutoff of 200 base pairs (bp). Contigs shorter than 200 bp were disregarded for this analysis. (B) Translated contigs length distribution. The vertical dashed line shows the arbitrary cutoff of 50 amino acids (aa). Contigs shorter than 50 aa were disregarded for this analysis. 

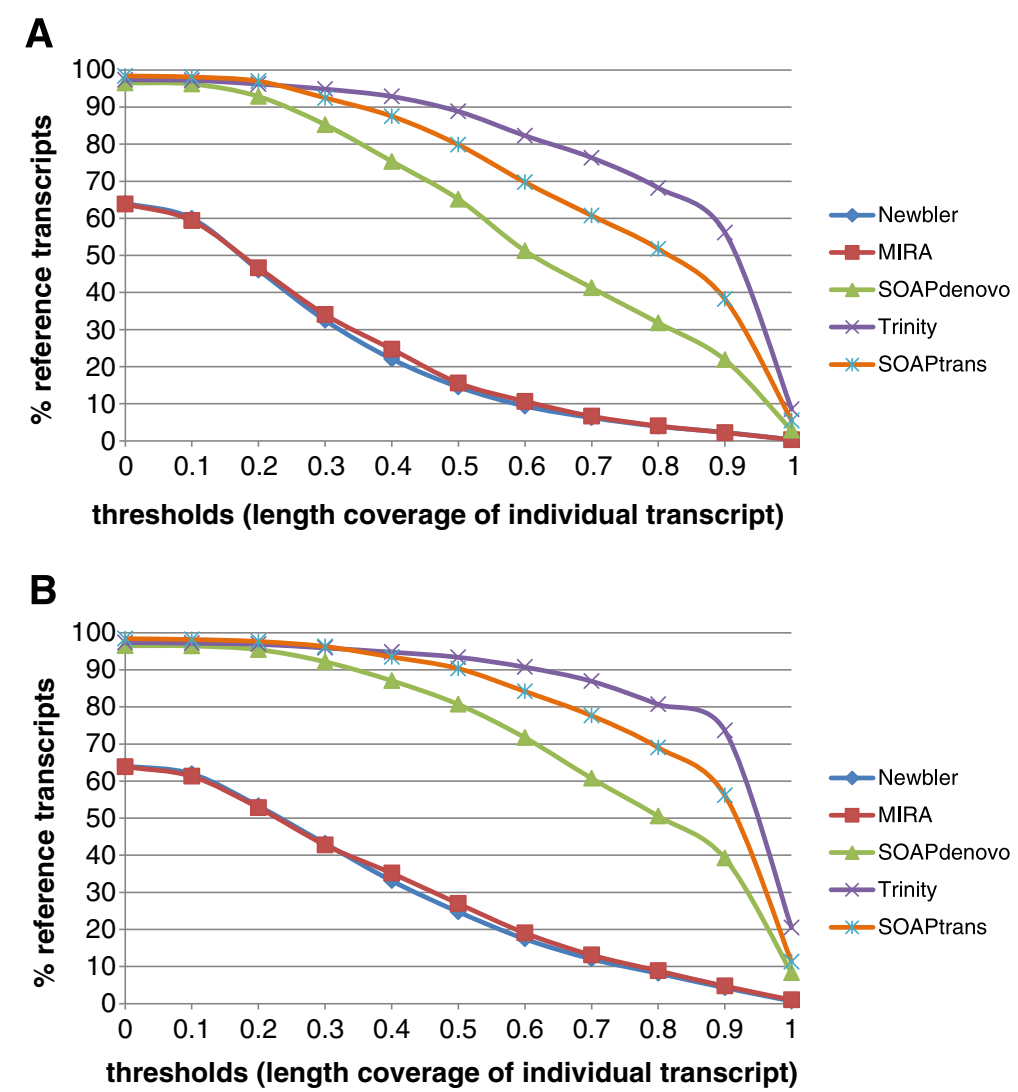

Figure 2 Contiguity (A) and completeness (B) of different assemblers at different thresholds. The assemblies were aligned with two published reference data bases: 357 ultra-conserved ortholog (UCO) coding sequence [35] and 959 single copy nuclear genes [36].

the categories cellular component, molecular function, and biological process were very similar (Figure 6). This similarity persists even though only emergent leaves were sampled for G. maderense versus four tissue types (emergent and expanded leaves, roots and flowers) for P. x hortorum.

To more directly address the question whether sequencing from multiple tissue types improves the breadth of transcriptome coverage, orthologous genes between $G$. maderense and A. thaliana and between $P$. x hortorum and $A$. thaliana were identified. Of the 35,386 protein sequences from $A$. thaliana, the G. maderense assembly had homologs to 11,131 sequences and the $P . \mathrm{x}$ hortorum assembly had homologs to 11,583 sequences. The comparable numbers of orthologous genes found for the two Geraniaceae species indicated that there was little improvement on the breadth of transcriptome coverage by sequencing from multiple tissue types ( 1 versus 4 tissues for G. maderense and P. x hortorum, respectively).

\section{Identification of selected organelle targeted genes}

Pentatricopeptide repeat proteins (PPRs) are a large family of RNA binding proteins encoded by over 400 genes in angiosperms; most are organelle targeted and involved in regulating organelle gene expression. The transcriptomes of $P . x$ hortorum and G. maderense were annotated using 429 Arabidopsis PPR sequences as a reference database (Table 5). The overall number of PPR genes varied considerably between the two Geraniaceae and Arabidopsis, with PPR gene number reduced in $P$. x hortorum. The numbers of P class PPR genes were found to be similar in all three species, whereas many fewer PLS class genes were found in the Geraniaceae, especially in $P . \mathrm{x}$ hortorum.

Sigma factors are nuclear encoded, plastid targeted proteins that assemble with four plastid encoded proteins (rpoA, rpoB, rpoC1 and rpoC2) to form the core of the PEP holoenzyme. At least one copy of each of the six Arabidopsis sigma factors was detected in both the $G$. maderense and P. $x$ hortorum transcriptomes (Table 5). The nucleotide and amino acid sequence identities between Arabidopsis/Geranium and Arabidopsis/Pelargonium for all six sigma factors were very similar (Table 6). The four contigs from G. maderense that aligned to sigma factor 2 were similar to each other in nucleotide sequence identity (87\%), suggesting that they may represent variant copies of the same gene. Two of the three contigs from $G$. maderense that aligned to sigma factor 5 were very similar to each other but less so to the third contig (98\% versus 


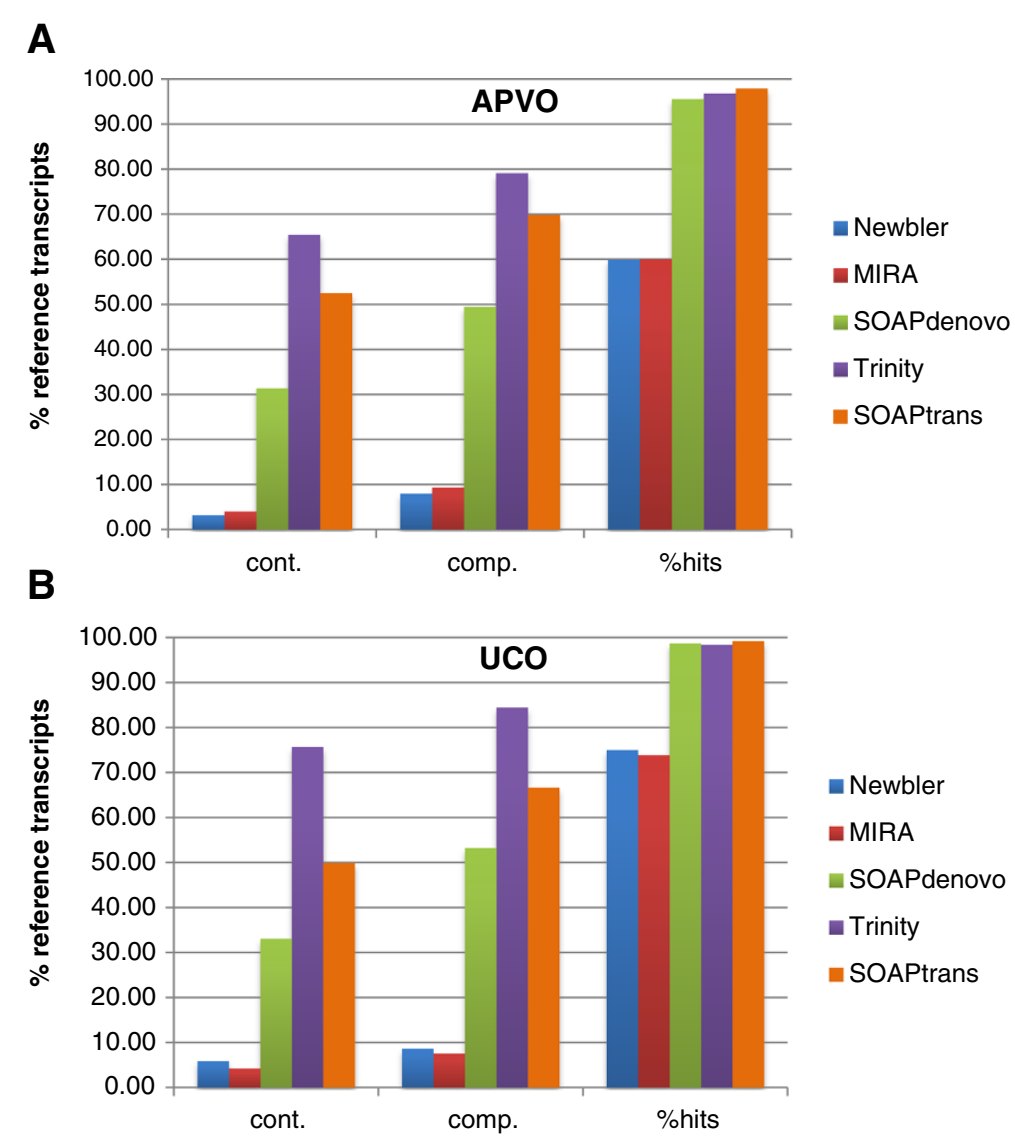

Figure $\mathbf{3}$ Completeness and contiguity results at threshold $\mathbf{8 0} \%$ using two published reference protein sets. Data sets: (A) 959 single copy nuclear genes (APVO); (B) 357 ultra-conserved ortholog (UCO) coding sequence [35,36]. Cont = contiguity, comp = completeness, \% hits = percentage of hits in reference transcriptome.

$71 \%$ nucleotide sequence identity). Sigma factors 2 and 6 were each represented by two $P$. $\mathrm{x}$ hortorum contigs, however only one of the contigs for each sigma factor appeared functional having start/stop codons at the $5^{\prime}$ and $3^{\prime}$ ends and lacking internal stop codons. Further experiments are needed to determine if the copies with internal stop codons are pseudogenes or assembly artifacts.

\section{Discussion}

\section{Strategies for de novo assembly of transcriptomes}

The use of NGS platforms is widespread and is applied in many research fields as volumes of data can be generated rapidly at a low cost relative to traditional Sanger sequencing [21]. RNA-seq, one popular NGS application, provides an efficient and cost-effective way of obtaining transcriptome data. There are a number of platforms available for generating NGS data $[37,38]$. Currently among the most popular are the Roche/454 FLX (http://www. roche.com) and the Illumina Hiseq 2000 (formerly Solexa; http://www.illumina.com) platforms. The Roche/454 FLX system is advantageous when longer reads are important (average read length $700 \mathrm{bp}$ ), whereas the Illumina system provides deeper sequencing coverage at a reduced cost per base, albeit with shorter read length (average length $100 \mathrm{bp}$ ).

For each platform various assemblers have emerged but during the past several years Roche 454 sequencing and the platform-specific assembler Newbler has been the most common approach for de novo assembly of transcriptome data [39-43]. This may be attributed to the idea that longer reads are more likely to overcome the specific challenges of de novo transcriptome assembly. Illumina sequencing has been used mainly when a related organism's genome was available for reference-based assembly [44,45], although due to recently increased read length it is becoming more common for use in de novo assembly as well [46,47]. Several recent studies compared the performance of different sequencing platforms and assembly methods [48-50] but none of these comparisons evaluated the level of completeness or contiguity of their assemblies, nor was the performance of the assemblers evaluated without known genome information, which is the situation for any project on non-model organisms. 


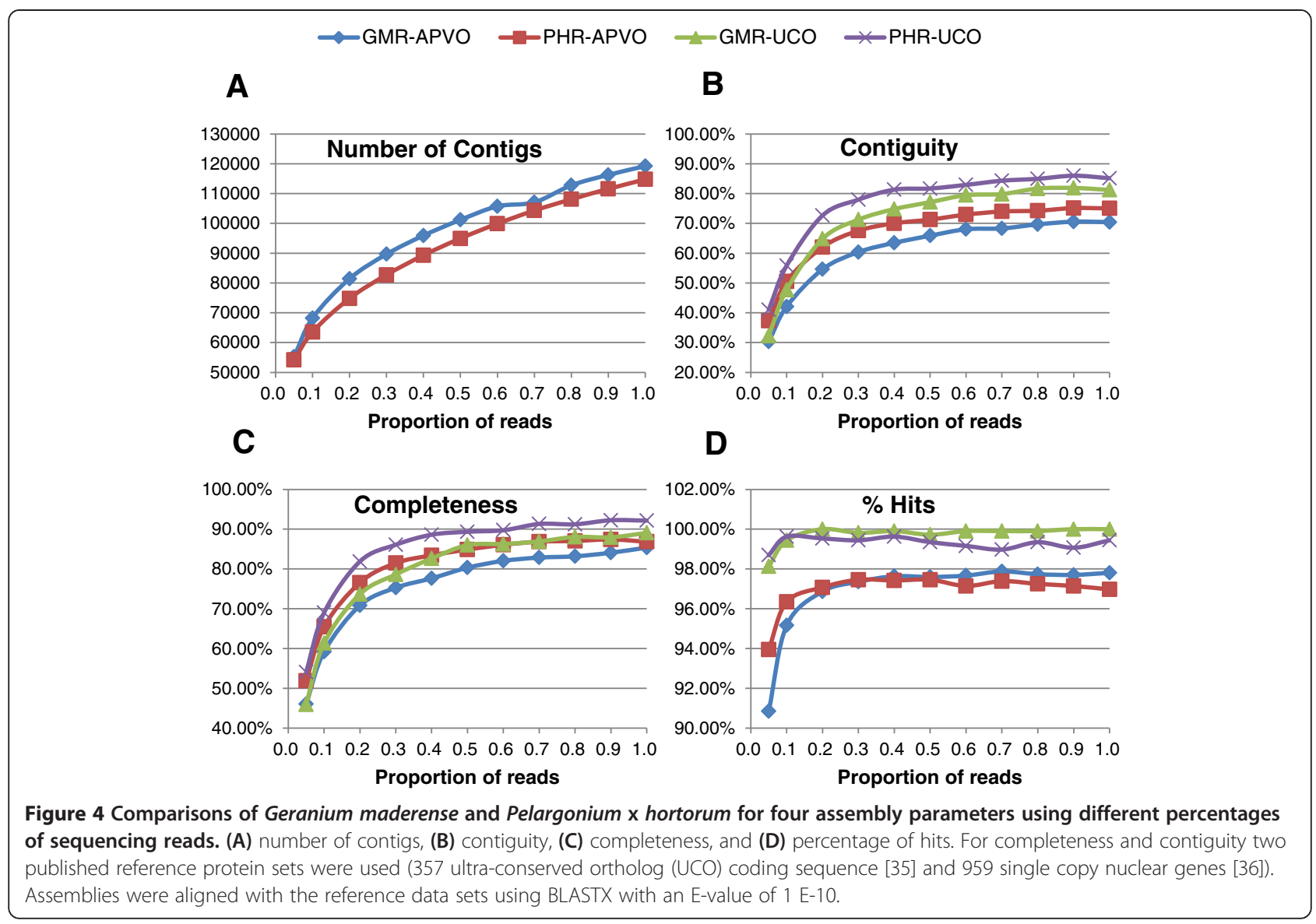

Our comparisons of sequencing platforms and assemblers for the Geraniaceae clearly indicated that the Illumina platform with Trinity assembly delivered the best performance in assembling a complete transcriptome in the absence of a reference genome. The Illumina assemblers (Trinity, SOAPdenovo, SOAPtrans) generated more contigs containing a greater total number of bases than the Roche/454 FLX assemblers (Newbler, MIRA). hile the MIRA assembly generated many more long contigs $(>6 \mathrm{~kb}$ ) than SOAPdenovo, the Trinity assembly out-performed all others in delivering long contigs, suggesting that the Trinity assembly contained more useful information than any of the other assemblies analyzed. While the Roche/454 FLX assemblies and the Illumina SOAPdenovo assembly produced similar results with regard to completeness and contiguity, the Illumina Trinity and SOAPtrans assemblies obtained much higher values for both parameters indicating that these assemblies comprise many more nearly complete transcripts (Figures 2 and 3).

\section{Functional annotation of Geraniaceae transcriptomes}

A total of 58,461 (49\%) and 42,506 (37\%) contigs were annotated from G. maderense and P. x hortorum, respectively. The low percentage of annotated contigs is most likely due to the large number of total contigs assembled. The number of aligned and annotated contigs is comparable to nine other recently published transcriptomes [22,27,51-56]. The number of annotated contigs in assemblies from both Geraniaceae species was very similar for the three major categories cellular component, molecular function, and biological process (Figure 6). This is encouraging since different tissues were sampled for the two species; only one tissue, emergent leaves for Geranium and four tissues, emergent leaves, expanded leaves, roots and flowers for Pelargonium. Particularly noteworthy is the detection of genes associated with flower and embryo development and pollen-pistil interaction since flowers were not sampled for Geranium. Overall, this comparison indicates that there is no marked improvement in transcriptome breadth of coverage when sampling four tissues compared to only emergent leaves.

\section{PPR proteins and sigma factors in Geraniaceae}

PPRs are a large family of RNA binding proteins encoded by over 450 genes in sequenced angiosperms. Most are organelle targeted and involved in regulating organelle gene expression [57]. Of the two classes (P and PLS) within the PPR family, those from PLS class (E and DYW subclasses) have been reported to be involved in RNA editing [14-16,58-64]. Previous studies have demonstrated 

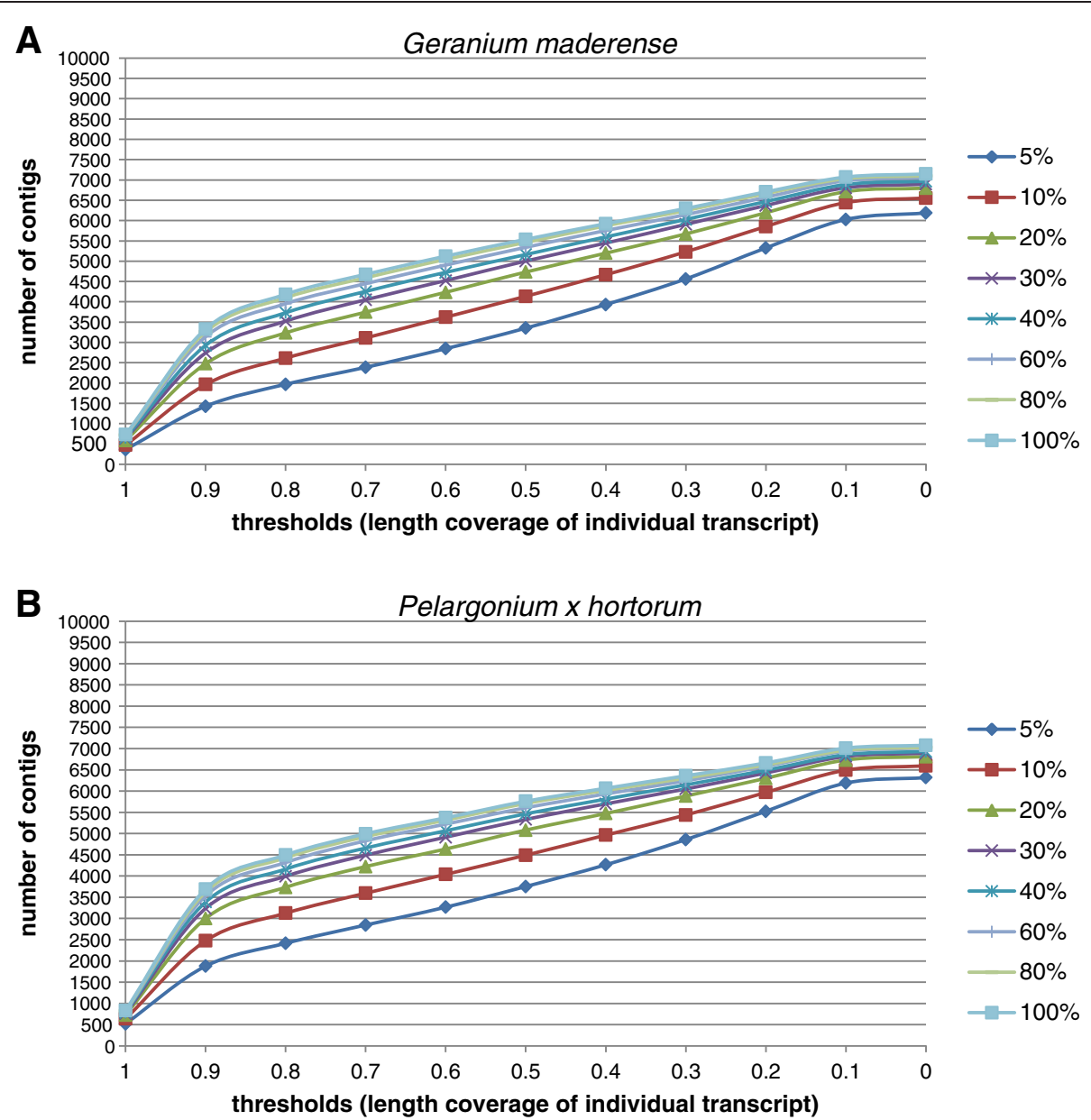

Figure 5 Contiguity of Geranium maderense (A) and Pelargonium $x$ hortorum (B) at different threshold values with differ percentages of reads using all Arabidopsis proteins from Uniprot/Swissprot database [85]. Assemblies were aligned with the database using BLASTX with an evalue of $1 \mathrm{E}-10$.

correlated evolution of PLS genes and RNA editing sites in plants $[17,65]$. Consistent with these results, a reduction in PLS genes (Table 5) in Geraniaceae was detected, where reduced editing frequency was previously demonstrated [1]. The reduced editing frequency and reduced PPR content in Geraniaceae is especially intriguing with respect to the increased mitochondrial substitution rate in this

Table 4 Statistics of transcriptome annotations for Geranium maderense (GMR) and Pelargonium $x$ hortorum (PHR)

\begin{tabular}{lll}
\hline & GMR & PHR \\
\hline Total contigs & 119,217 & 114,762 \\
Aligned contigs & 76,332 & 56,283 \\
Annotated contigs & 58,461 & 42,506 \\
Assigned GO terms & 311,108 & 222,765 \\
Assigned EC & 25,533 & 19,354 \\
Contigs with EC & 20,337 & 15,252 \\
\hline
\end{tabular}

$\mathrm{GO}=$ Gene Ontology; $\mathrm{EC}=$ Enzyme Code. family. Although an inverse correlation between editing frequency and substitution rate has been noted previously in Geraniaceae and other taxa $[1,66-68]$, the finding that PPR gene content is also reduced in Geraniaceae indicates that this family is ideally suited for future studies assessing the evolutionary dynamics of editing frequency, PPR content, and mitochondrial substitution rates.

One long-standing question regarding the plastid genomes in Geraniaceae is the putative loss of the rpo $A$ gene from $P . \mathrm{x}$ hortorum [69-71]. The complete plastid genome sequence of this species revealed several rpoA-like open reading frames (ORFs) that are highly divergent relative to rpoA genes in other angiosperms or even other Geraniaceae [11,12]. Two alternative explanations were suggested for these observations: (1) a copy of the gene in the nucleus had gained functionality; or (2) at least one of the highly divergent $r p o A$-like ORFs remains functional. Extensive evolutionary rate comparisons of plastid genes across the Geraniaceae revealed that the other three PEP subunits (rpoB, rpoC1, rpoC2) have 


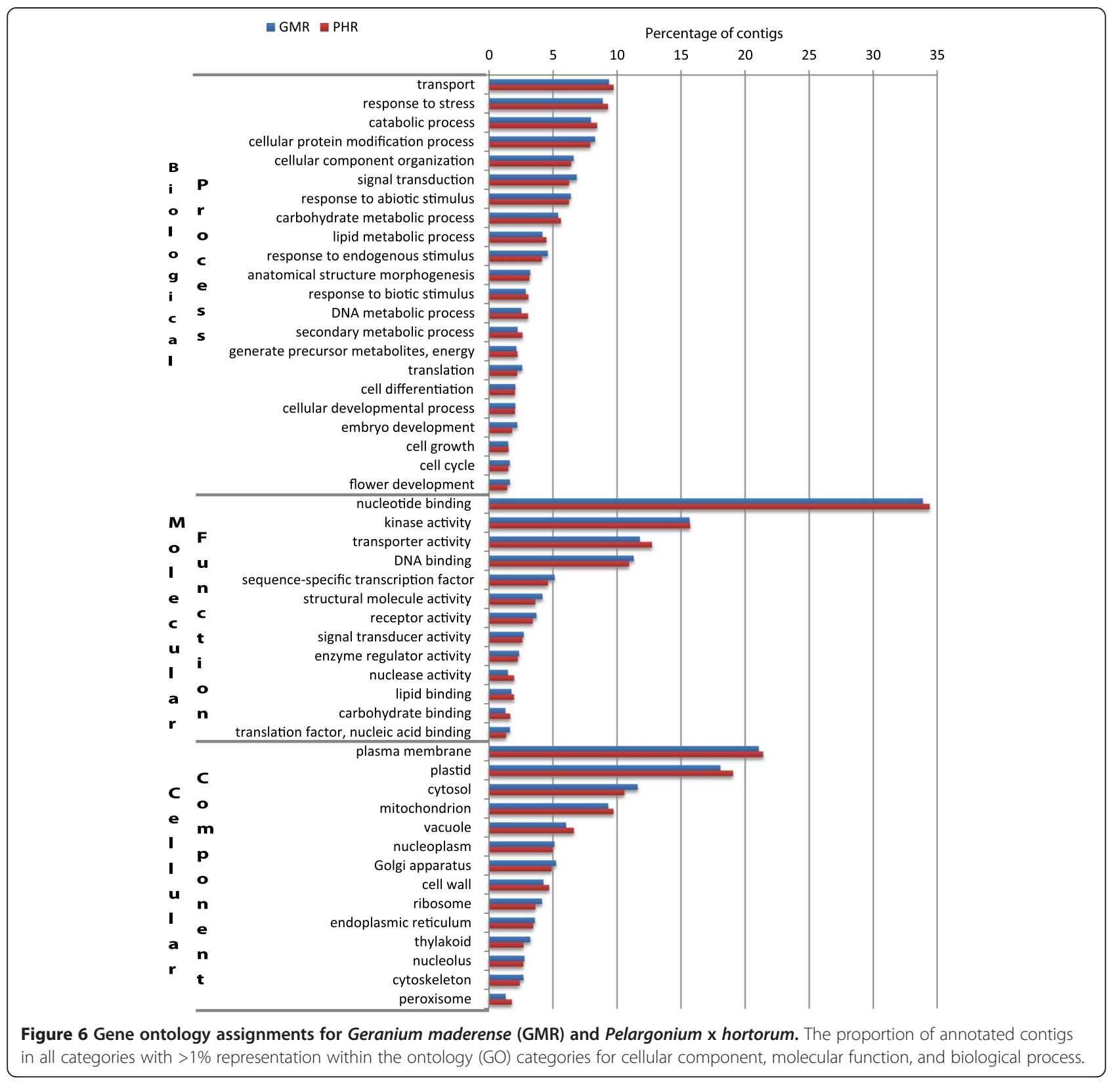

significantly elevated nucleotide substitution rates and have likely experienced positive selection $[8,9]$. Despite exhaustive searching of the nuclear transcriptome of $P$. x hortorum no copy of the rpoA gene was detected. However, intact copies of all six sigma factors, which are required for PEP to function [72], were identified in the transcriptome. The holoparasite Phelipanche aegyptiaca lacks a functional PEP and mining unigene files published in a recent transcriptomic study of parasitic plants [20] failed to uncover a single sigma factor suggesting that in species where PEP sequences are lost from the plastid the requisite sigma factors are also absent from the nuclear transcriptome. The identification of all six sigma factors in the $P . \mathrm{x}$ hortorum transcriptome supports the likelihood that PEP is active in P. $\mathrm{x}$ hortorum plastids.

\section{Conclusions}

With the widespread application of NGS techniques, the ability to process and analyze massive quantities of sequence data in a timely manner becomes imperative to a successful project. Regardless of the goals of a particular project, it is desirable to obtain data that is as accurate and complete as possible in a way that is cost effective as well as timely. In this study a cross-platform comparison of de novo transcriptome assembly was conducted using representative species from the two largest genera of 
Table 5 PPR protein ${ }^{\mathrm{a}}$ and sigma factor ${ }^{\mathrm{b}}$ distribution

\begin{tabular}{llll}
\hline & $\begin{array}{l}\text { Arabidopsis } \\
\text { thaliana }\end{array}$ & $\begin{array}{l}\text { Geranium } \\
\text { maderense }\end{array}$ & $\begin{array}{l}\text { Pelargonium } \\
\text { hortorum }\end{array}$ \\
\hline PPR proteins & 429 & 523 & 315 \\
P class & 238 & 387 & 262 \\
PLS-E class & 105 & 96 & 22 \\
PLS-DYW class & 86 & 40 & 31 \\
Sigma factors & 6 & 10 & 6 \\
Sig 1 & 1 & 1 & 1 \\
Sig 2 & 1 & 4 & 1 \\
Sig 3 & 1 & 1 & 1 \\
Sig 4 & 1 & 1 & 1 \\
Sig 5 & 1 & 3 & 1 \\
Sig 6 & 1 & 1 & 1 \\
\hline
\end{tabular}

aPR protein data of Arabidopsis are from Small and Peeters [92]. The PPR class represents the number contigs longer than 150 aa, which is the minimum length of PPR proteins identified in Arabidopsis. ${ }^{\text {b}}$ The number of total contigs and the number of intact contigs aligned to the reference sigma factors are shown. Intact contigs are those with start/stop codons on 5' and $3^{\prime}$ ends, and without any internal stop codons.

Geraniaceae, G. maderense and P. x hortorum. As no reference genome is available for Geraniaceae, or any of its close relatives, this approach represents a truly de novo assembly allowing evaluation of efficacy among the platforms/assemblers that more closely resembles current NGS research. The assembly of Illumina Hiseq 2000 reads with Trinity or SOAPtrans was highly effective in reconstructing, as completely as is currently feasible, the protein-coding transcripts of Geraniaceae. As for the differences between the two assemblers, Trinity generated slightly more single contiguous contigs and reconstructed more reference genes with a combination of multiple contigs, while SOAPtrans ran much faster than Trinity. These differences in contiguity and completeness became more obvious with the reduced set of input data $\left(1 / 40^{\text {th }}\right.$ in this case). These findings recommend the Illumina platform with Trinity assembly to obtain the most complete gene coverage by a single contig, especially when a small amount of reads are available. In instances where a large amount of data is available and there are limited computational resources, Illumina SOAPtrans assembly may be preferred as it generated a relatively complete assembly much more quickly than Trinity. Furthermore, evaluation of the amount of Illumina sequence data required for generating a complete transcriptome is approximately 60 million reads.

Geraniaceae organelle genomes have been shown to exhibit a number of unusual features relative to other angiosperms, including highly accelerated rates of nucleotide substitutions in both mitochondrial and plastid genes $[1,8,9]$, reduced RNA editing in mitochondrial genomes [1] and highly rearranged plastid genomes [10-13]. This comparative transcriptome analysis of $G$. maderense and $P . \times$ hortorum detected a reduction in PPR proteins associated with RNA editing, which corresponds with reduced RNA editing in the mitochondria. Examination of nuclear encoded, plastid targeted sigma factors required for PEP function supports the hypothesis that PEP is active in P. $\mathrm{x}$ hortorum plastids, possibly incorporating the product of at least one of the highly divergent rpoA-like ORFs in the plastid genome.

\section{Methods}

\section{RNA isolation}

Plant tissues were collected from live plants grown in the University of Texas (UT) greenhouse and frozen in liquid nitrogen for two species from different genera of Geraniaceae, Geranium maderense and Pelargonium x hortorum cv ringo white. For Pelargonium leaf and inflorescence samples were collected. Leaves were of two developmental stages, newly emerged and fully expanded. Entire inflorescences were harvested prior to anthesis. Root samples of $P . x$ hortorum were harvested from specimens grown aseptically in agar media. For $\mathrm{Ge}$ ranium, only emergent leaves were collected. Total RNA was isolated separately from each sample type by grinding in liquid nitrogen followed by $30 \mathrm{~min}$ incubation at $65^{\circ} \mathrm{C}$ in two volumes of extraction buffer (2\% Cetyltrimethylammonium bromide, 3\% Polyvinylpyrrolidone-40, 3\% 2Mercaptoethanol, $25 \mathrm{mM}$ Ethylenediaminetetraacetic acid, $100 \mathrm{mM}$ Tris(hydroxymethyl)aminomethane- $\mathrm{HCl} \mathrm{pH} 8$, $2 \mathrm{M} \mathrm{NaCl}, 2.5 \mathrm{mM}$ spermidine trihydrochloride) with vortexing at $5 \mathrm{~min}$ intervals. Phase separation with

Table 6 Sequence identities between intact contigs in Geraniaceae and Arabidopsis thaliana sigma factors

\begin{tabular}{|c|c|c|c|c|}
\hline \multirow{2}{*}{$\begin{array}{l}\text { Arabidopsis thaliana } \\
\text { Sequence identity (\%) }\end{array}$} & \multicolumn{2}{|c|}{ Geranium maderense } & \multicolumn{2}{|c|}{ Pelargonium $\mathrm{x}$ hortorum } \\
\hline & Nucleotide & Amino acid & Nucleotide & Amino acid \\
\hline Sig 1 & 64.5 & 52.4 & 61.1 & 50.4 \\
\hline $\operatorname{sig} 2$ & 62.6 & 48.9 & 62.5 & 47.1 \\
\hline Sig 3 & 57.7 & 39.9 & 58.6 & 43.3 \\
\hline $\operatorname{Sig} 4$ & 58.6 & 42.6 & 58.3 & 43.5 \\
\hline $\operatorname{Sig} 5$ & 64.3 & 54.1 & 65.8 & 55.0 \\
\hline Sig 6 & 58.6 & 41.1 & 59.6 & 42.4 \\
\hline
\end{tabular}

an cases where there is more than one intact contig for a sigma factor, the one with highest sequence identity to Arabidopsis was selected for comparison. 
chloroform:isolamyl alcohol (24:1) was performed twice and the aqueous phase was adjusted to $2 \mathrm{M} \mathrm{LiCl}$. Samples were precipitated overnight at $4{ }^{\circ} \mathrm{C}$ and total RNA was pelleted by centrifugation at $17,000 \times \mathrm{g}$ for $20 \mathrm{~min}$ at $4^{\circ} \mathrm{C}$. RNA pellets were washed once with $70 \%$ ethanol and air dried at room temperature. Following resuspension in RNase free water, RNAs were analyzed by denaturing gel electrophoresis and by spectrophotometry. For Pelargonium, the four tissue types were pooled in equimolar ratio. All RNAs were treated with DNase I (Fermentas, Glen Burnie MD, USA) according to the product protocol. DNase I was removed from the solution by extraction with phenol:chloroform:isoamyl alcohol (25:24:1) and the aqueous phase was adjusted to $0.3 \mathrm{M}$ sodium acetate. RNA was precipitated with 2.5 volumes of cold absolute ethanol for $20 \mathrm{~min}$ at $-80^{\circ} \mathrm{C}$. Pellets were washed with $70 \%$ ethanol, air-dried and resuspended in water to $1 \mu \mathrm{g} \mu \mathrm{L}^{-1}$. Total RNA sample aliquots were frozen in liquid nitrogen and shipped on dry ice to the Beijing Genomics Institute (BGI) in Hong Kong or delivered to the Genome Sequencing Analysis Facility (GSAF) at UT. Confirmation of sample quality and concentration was conducted at each facility using the Agilent 2100 Bioanalyzer instrument (Agilent Technologies, Santa Clara CA, USA).

\section{Illumina sequencing}

Sample preparation for Illumina sequencing was performed at BGI according to Illumina's protocol (Part \# 1004898 Rev. D). Total RNA was treated with the Ribo-Zero ${ }^{\mathrm{rm}} \mathrm{rRNA}$ Removal Kit (Epicentre Biotechnolgies, Madison WI, USA) prior to fragmentation and priming with random hexamers for first strand cDNA synthesis using SuperScript ${ }^{\circ}$ III Reverse Transcriptase (Invitrogen, Beijing, China). Second strand cDNA synthesis was carried out using RNase $\mathrm{H}$ (Invitrogen) and DNA polymerase I (New England BioLabs, Beijing, China). The resulting cDNA fragments were purified with QIAQuick ${ }^{\circ}$ PCR extraction kit (Qiagen, Shanghai, China) and normalized with Duplex-Specific thermostable nuclease (DSN) enzyme from Kamchatka crab (Evrogen, Moscow, Russia) according to the protocol outlined by Invitrogen (Part \# 15014673 Rev. C). End repair and adenylation of the normalized cDNA library was followed by ligation to the paired-end (PE) sequencing adapters. Following gel electrophoresis for size selection (180-220 bp) the library was PCR amplified for sequencing using the Illumina HiSeq ${ }^{\text {mix }} 2000$. The PE library was sequenced for $101 \mathrm{bp}$.

\section{Roche/454 FLX sequencing}

The method for cDNA library construction and normalization was based on that of Meyer et al. [73]. Briefly, total RNA was reverse-transcribed using oligo-dT coupled to a PCR-suppression primer. The reverse complement of this primer was incorporated at the $3^{\prime}$ end of the first-strand
cDNA using the template switching capability of the SuperScript II Reverse Transcriptase (Invitrogen). Duplexspecific nuclease was added to digest the abundant double-stranded cDNA. After purification, PCR was performed, and the product was purified and sheared by nebulization. The fragmented DNA was then endrepaired and ligated to Roche Rapid library adaptors using the NEBNext ${ }^{\circ}$ Quick DNA Sample Prep Master Mix Set 2 and NEBNext ${ }^{\circ}$ DNA Sample Prep Master Mix Set 2 (New England BioLabs). Final library size and concentration were measured on the Agilent BioAnalyzer and by qPCR before sequencing on the Roche/454 FLX sequencer.

\section{Read pre-processing}

Raw reads were preprocessed to eliminate contaminant and low quality sequences. Filtering of Illumina Hiseq 2000 reads included the removal of low quality bases, reads where (poly) adenosine constitutes more than $6 \%$ of bases, and reads containing specialized features such as adaptors and other artifacts arising from library construction. Roche/454 FLX reads were preprocessed by removing reads shorter than $50 \mathrm{bp}$ and reads with artificial sequences based on a vector reference file. The complete data set is available at NCBI Sequence Read Archive (Accession numbers SRA059171 for Geranium and SRA053016.1 for Pelargonium).

\section{Ribosomal RNA content and Illumina library complexity}

Ribosomal RNA (rRNA) contigs were identified using reciprocal blast of rRNA from Arabidopsis (5.8S, $18 \mathrm{~S}$ and $25 \mathrm{~S}$ from nucleus, $5 \mathrm{~S}, 16 \mathrm{~S}$ and $23 \mathrm{~S}$ in chloroplast, and $5 \mathrm{~S}, 18 \mathrm{~S}$ and $26 \mathrm{~S}$ in mitochondria) as reference. The rRNA sequences from Arabidopsis were downloaded from TAIR [74]. Ribosomal RNA reads were removed prior to the library complexity analysis. Due to a lack of nuclear genome sequence, the remaining reads were mapped back to the whole transcriptome data using bowtie 2 [75]. The mapping results were sorted using samtools [76] and then analyzed with MarkDuplicates module of Picard [32].

\section{Assembly}

Transcritpome assemblies were initially performed on Pelargonium using a variety of assemblers to compare the efficacy of different platforms and assemblers. After these initial comparisons, all subsequent assembles were performed on both Geranium and Pelargonium using Trinity and Illumina data. For assembly of clean Illumina reads, Trinity [77], SOAPdenovo and SOAPtrans (http://soap.genomics.org.cn/SOAPdenovo-Trans.html) [78,79] were used. Trinity, released on 2011-08-20 (http://sourceforge.net/projects/trinityrnaseq/), was run with parameters "-seqType fq -CPU 10 -paired_fragment_length 200 -run_butterfly” on 
a 24-core $3.33 \mathrm{GHz}$ linux work station with $1 \mathrm{~TB}$ memory at the Texas Advanced Computing Center (TACC, http:// www.tacc.utexas.edu/). The assembly was split into three steps according to the provided script trinity.pl released with the software. The split scripts run the corresponding three steps in Trinity: inchworm, chrysalis, and butterfly. The parameters were the same for each step, and each step picked up the previous step's output as input and processed it. The scripts will be provided by JZ upon request. The SOAPtrans assembly was run with the parameters "kmer $=61$, max_rd_length $=100$, avg_ins $=200$ " on the same server as that of Trinity. For SOAPtrans kmer lengths from 23 bp to 81 bp were explored; 61 bp was selected because it generated the best contiguity compared with other kmer values. The SOAPdenovo assembly was done at BGI on a 48-core $2.67 \mathrm{GHz}$ Linux workstation with $50 \mathrm{~GB}$ memory with parameters "Kmer $=41$, insert size $=200$, overlap threshold $=50$ " for assembly, and "Kmer +1 " to fill the gaps. The generated fasta file was postprocessed by BGI to remove the sequences shorter than 150 bp. Assembly of Roche/454 FLX utilized MIRA [80] and Newbler [81]. MIRA 3.4.0 for a 64-bit linux system (http://sourceforge.net/projects/mira-assembler/ files/MIRA/stable/) was released on 2011-08-21. MIRA was run with parameters "-job=denovo, est, accurate, 454 -fasta 454_SETTINGS” on a 12-core $3.33 \mathrm{GHz}$ linux work station with 24 GB memory at TACC. Newbler 2.6 accompanies the Roche/454 FLX platform and assembly was conducted at UT GSAF on 24-core $2.40 \mathrm{GHz}$ linux work station with $64 \mathrm{~GB}$ memory using the parameters "runAssembly -cpu 8 -urt -cdna -vt vector.fa".

\section{Comparative analysis of assemblies}

Trinity, SOAPdenovo and SOAPtrans assembly output comprised a single contig file each and these were used in the analyses. Unpadded fasta files were selected from the MIRA output and the isotig file was selected from the Newbler output for use in analyses.

The initial assembly quality was evaluated using the following metrics: number of assembled contigs, maximum, minimum and mean contig length, N50 and redundancy. Initial assembly statistics and contig length distribution analysis was done by custom perl scripts and MATLAB version R2011b. Contig clustering and removal of redundant contig sequences was performed using CD-HIT [82]. CD-HIT version 4.5.4 (downloaded from http://code. google.com/p/cdhit/downloads/list) was executed using parameters "cd-hit -c 1.0 -n 5 -T 12" for cDNA sequences and "cd-hit-est -c 1.0 -n 10 -T 12" for protein sequences. Redundancy was calculated from the difference between the number of contigs before and after clustering. Maximum, minimum, and mean contig length, N50 and total bases were calculated from the contigs after clustering and removal of those contigs $<200 \mathrm{bp}$.
The assemblies were aligned to two published reference databases: 357 ultra-conserved ortholog (UCO) coding sequence [35] from Arabidopsis (sequences available at: http://compgenomics.ucdavis.edu/compositae_reference. php), and a list of 959 single copy nuclear genes shared between Arabidopsis, Oryza, Populus, and Vitis [36] using BLASTX with evalue of $1 \mathrm{E}-10$. Contig alignment to the reference databases utilized the standalone BLAST + [83] program for 64-bit linux system (ftp://ftp.ncbi.nlm.nih.gov/ blast/executables/blast+/LATEST/). The parameters for BLAST + DNA alignment were "blastn -task blastn -evalue 1 E-10 -word_size 11 -outfmt 6 -num_threads 12". Parameters for protein alignment were "blastp -task blastp -num_threads 12 -outfmt 6". For blastp, two different e values were used, 1 E-10 and 1 E-20, in order to address the generality of the results. Multiple sequence alignment was done by muscle [84]. Muscle for 64-bit linux system (http://www.drive5.com/muscle/ downloads.htm) was used with default parameters.

The local reference database for identifying the open reading frames contained four proteomes downloaded from Phytozome (http://www.phytozome.net/search.php): Citrus clementina, C. sinensis, Eucalyptus grandis and Arabidopsis thaliana. Contigs were translated by alignment to the local database using blastx to identify open reading frames. The blastx parameter was "blastx -evalue 1e-6 -max_target_Seqs 1 -num_threads 48 -outfmt'6 std qframe”. The reading frame parameter was added to the output in order to facilitate the following analysis. The aligned regions of contigs were translated, extracted, and then extended by translating the contigs in both directions according to standard codon usage until a stop codon was encountered. The translated contigs were clustered again using CD-HIT at a threshold of $100 \%$ and all other parameters used the default settings. Two parameters, contiguity and completeness as described by Martin and Wang [85] were used to evaluate the alignment results. Briefly, contiguity is defined as the percentage of the reference transcripts covered at some arbitrary coverage threshold by a single longest contig. Completeness is defined as the percentage of the reference transcripts covered at a threshold by multiple assembled contigs (Box one in [85]). In this study a range of thresholds up to $100 \%$ was evaluated, and $80 \%$ was selected as the threshold for both contiguity and completeness calculations. Both parameters were calculated with protein sequence alignment, and the alignment results were analyzed using custom perl scripts available from JZ upon request.

\section{Evaluation of assemblies with different proportion of reads}

To assess how much data (number of reads) is needed to construct the complete transcriptome, different proportions of sequencing data ranging from $5 \%$ to $100 \%$ were extracted for both species. The extracted reads were assembled with Trinity using the parameters described 
above. Extraction and assembly were repeated three times for each proportion except $100 \%$, and the assembly statistics (contig number, contiguity and etc.) were averaged.

Basic statistics and assembly parameters such as contiguity and completeness were calculated using the same local database described above. To determine how well the assemblies cover a complete transcriptome, the custom Arabidopsis protein database was constructed by extracting all Arabidopsis proteins from Uniprot/Swissprot database [86], and protein sequences with name "hypothetical" or "predicted" were discarded. The assemblies were aligned with the database using BLASTX with an E-value of 1 E-10.

\section{Orthologous genes identification}

Orthologous genes between transcriptomes of G. maderense, $P . \mathrm{x}$ hortorum and $A$. thaliana were identified with reciprocal blast with parameters "blastp -task blastp -num threads 12 -max_target_Seqs 1 -evalue 1e-10 -outfmt = '6 std qlen slen". Blast results were analyzed with custom perl scripts.

\section{Functional annotation}

The assemblies were aligned with the NCBI nr database using BLASTX with an E-value of 1 E- 6 and taking the best 10 hits for annotation. The blast results were used to annotate each sequence with gene ontology (GO) terms using Blast2GO [87-89]. To improve the efficiency of annotation, local blast2go database was downloaded (http:// www.blast2go.com/b2glaunch/resources/35-localb2gdb). GO terms were mapped to the reduced GO-slim (plant) ontology to get a broader functional representation of the transcriptome.

\section{Identification of selected organelle targeted genes}

PPR proteins were searched for using HMMER [90,91] with previously established PPR motif alignment files [92]. Transcript sequences with more than one PPR motif were considered PPR genes. Sigma factor protein sequences from Arabidopsis were downloaded from TAIR [74] and used as reference. Sigma factor structure and conserved domain information were obtained from previous studies [93-95]. Putative transit peptides were predicted with targetP $[96,97]$. Orthologs from two transcriptomes of G. maderense and $P$. x hortorum were identified by reciprocal blast at E-value $1 \mathrm{E}-10$.

\section{Additional files}

Additional file 1: Contiguity and completeness of different protein data sets at E-value $1 \mathrm{E}-10\left(1 / 40^{\text {th }}\right.$ of the Illumina data was used by Trinity).

Additional file 2: Transcriptome annotation for Geranium maderense and Pelargonium $\mathrm{x}$ hortorum.

\section{Competing interests}

The authors declare that they have no competing interests.

\section{Authors' contributions}

JZ contributed to the design of the project, performed all analyses and drafted the manuscript; TAR isolated RNA, drafted RNA and sequencing methods sections, contributed to the design of the project, and assisted with manuscript preparation; JPM contributed to the design of the project and assisted with manuscript preparation; RKJ contributed to the design of the project and assisted with manuscript preparation. All authors read and approved the final draft of the manuscript.

\section{Acknowledgements}

Support was provided by the National Science Foundation (IOS-1027259 to RJK, JPM and TAR). The authors thank Scott Hunicke-Smith, Ely Meyer, and Heather Deiderick at the Genome Sequencing and Analysis Facility at the University of Texas for assistance in 454 sequencing and for discussions about assembly of 454 transcriptome data, the Texas Advanced Computing Center (TACC) at the University of Texas for access to supercomputers, the Beijing Genomics Institute for Illumina sequencing, and Chris Blazier and Mao-Lun Weng for valuable comments on an earlier draft of the manuscript.

\section{Author details}

${ }^{1}$ Department of Integrative Biology and Institute of Cellular and Molecular Biology, The University of Texas at Austin, 205 W. 24th St. Stop C0930, Austin, TX 78712, USA. ${ }^{2}$ Center for Plant Science Innovation and Department of Agronomy and Horticulture, University of Nebraska-Lincoln, Lincoln, NE 68588, USA. ${ }^{3}$ Genomics and Biotechnology Section, Department of Biological Sciences, Faculty of Science, King Abdulaziz University, Jeddah 21589, Saudi Arabia.

Received: 23 May 2013 Accepted: 20 December 2013

Published: 29 December 2013

\section{References}

1. Parkinson CL, Mower JP, Qiu Y-Q, Shirk AJ, Song K, Young ND, dePamphilis CW, Palmer JD: Multiple major increases and decreases in mitochondrial substitution rates in the plant family Geraniaceae. BMC Evol Biol 2005, 5:73.

2. Bakker FT, Breman F, Merckx $V$ : DNA sequence evolution in fast evolving mitochondrial DNA nad1 exons in Geraniaceae and Plantaginaceae. Taxon 2006, 55:887-896.

3. Cho Y, Mower JP, Qiu YL, Palmer JD: Mitochondrial substitution rates are extraordinarily elevated and variable in a genus of flowering plants. Proc Natl Acad Sci U S A 2004, 101:17741-17746.

4. Mower JP, Touzet P, Gummow JS, Delph LS, Palmer JD: Extensive variation in synonymous substitution rates in mitochondrial genes of seed plants. BMC Evol Biol 2007, 7:135.

5. Sloan DB, Barr CM, Olson MS, Keller SR, Taylor DR: Evolutionary rate variation at multiple level of biological organization in plant mitochondrial DNA. Mol Biol Evol 2008, 25:243-246.

6. Sloan DB, Oxelman B, Rautenberg A, Taylor DR: Phylogenetic analysis of mitochondrial substitution rate variation in the angiosperm tribe Sileneae. BMC Evol Biol 2009, 9:260.

7. Adams KL, Qiu YL, Stoutemyer M, Palmer JD: Punctuated evolution of mitochondrial gene content: high and variable rates of mitochondrial gene loss and transfer during angiosperm evolution. Proc Natl Acad Sci U S A 2002, 99:9905-9912.

8. Guisinger MM, Kuehl JV, Boore JL, Jansen RK: Genome-wide analyses of Geraniaceae plastid DNA reveal unprecedented patterns of increased nucleotide substitutions. Proc Natl Acad Sci U S A 2008, 105:18424-18429.

9. Weng ML, Ruhlman TA, Gibby M, Jansen RK: Phylogeny, rate variation, and genome size evolution of Pelargonium (Geraniaceae). Mol Phylogen Evol 2012, 64:654-670.

10. Palmer JD, Nugent JM, Herbon LA: Unusual structure of geranium chloroplast DNA: a triple-sized inverted repeat, extensive gene duplications, multiple inversions and two repeat families. Proc Natl Acad Sci U S A 1987, 84:769-773.

11. Chumley TW, Palmer JD, Mower JP, Fourcade HM, Calie PJ, Boore JL, Jansen RK: The complete chloroplast genome sequence of Pelargonium $\mathrm{x}$ hortorum: organization and evolution of the largest and most highly rearranged chloroplast genome of land plants. Mol Biol Evol 2006, 23:2175-2190. 
12. Guisinger MM, Kuehl JV, Boore JL, Jansen RK: Extreme reconfiguration of plastid genomes in the angiosperm family Geraniaceae: rearrangements, repeats, and codon usage. Mol Biol Evol 2011, 28:583-600.

13. Blazier CJ, Guisinger MM, Jansen RK: Recent loss of plastid-encoded $n d h$ genes within Erodium (Geraniaceae). Plant Mol Biol 2011, 76:263-272.

14. Kotera $E$, Tasaka M, Shikanai T: A pentatricopeptide repeat protein is essential for RNA editing in chloroplasts. Nature 2005, 433:326-330.

15. Okuda K, Myouga F, Motohashi R, Shinozaki K, Shikanai T: Conserved domain structure of pentatricopeptide repeat proteins involved in chloroplast RNA editing. Proc Natl Acad Sci U S A 2007, 104:8178-8183.

16. Okuda K, Chateigner-Boutin AL, Nakamura T, Delannoy E, Sugita M, Myouga F, Motohashi R, Shinozaki K, Small I, Shikanai T: Pentatricopeptide repeat proteins with the DYW motif have distinct molecular functions in RNA editing and RNA cleavage in Arabidopsis chloroplasts. Plant Cell 2009, 21:146-156.

17. Fujii S, Small I: The evolution of RNA editing and pentatricopeptide repeat genes. New Phytol 2011, 191:37-47.

18. Sugiura C, Kobayashi Y, Aoki S, Sugita C, Sugita M: Complete chloroplast DNA sequence of the moss Physcomitrella patens: evidence for the loss and relocation of $r p o A$ from the chloroplast to the nucleus. Nucleic Acids Res 2003, 31:5324-5331.

19. Goffinet B, Wickett NJ, Shaw AJ, Cox CJ: Phylogenetic significance of the rpoA loss in the chloroplast genome of mosses. Taxon 2005, 54:353-360.

20. Wickett NJ, Honaas LA, Wafula EK, Das M, Huang K, Wu B, Landherr L, Timko MP, Yoder J, Westwood JH, dePamphilis CW: Transcriptomes of the parasitic plant family Orobanchaceae reveal surprising conservation of chlorophyll synthesis. Curr Biol 2011, 21:2098-2104.

21. Kircher M, Kelso J: High-throughput DNA sequencing-concepts and limitations. Bioessays 2010, 32:524-536.

22. Ward JA, Ponnala L, Weber CA: Strategies for transcriptome analysis in nonmodel plants. Amer J Bot 2012, 99:267-276.

23. Pepke S, Wold B, Mortazavi A: Computation for ChIP-seq and RNA-seq studies. Nat Methods 2009, 6:S22-S32

24. Wheat CW: Rapidly developing functional genomics in ecological model systems via 454 transcriptome sequencing. Genetica 2010, 138:433-451.

25. Der JP, Barker MS, Wickett NJ, dePamphilis CW, Wolf PG: De novo characterization of the gametophyte transcriptome in bracken fern, Pteridium aquilinum. BMC Genomics 2011, 12:99.

26. Barker MS, Vogel H, Schranz ME: Paleopolyploidy in the Brassicales: analyses of the Cleome transcriptome elucidate the history of genome duplications in Arabidopsis and other Brassicales. Genome Biol Evol 2009, 1:391-399.

27. Angeloni F, Wagemaker CA, Jetten MSM, Camp HJMO, Janssen-Megens EM, Francoijs KJ, Stunnenberg HG, Ouborg NJ: De novo transcriptome characterization and development of genomic tools for Scabiosa columbaria L. using next-generation sequencing techniques. Mol Ecol Resour 2011, 11:662-674.

28. Hou R, Bao Z, Wang S, Su H, Li Y, Du H, Hu J, Wang S, Hu X: Transcriptome sequencing and de novo analysis for Yesso scallop (Patinopecten yessoensis) using 454 GS FLX. PLoS One 2011, 6:e21560.

29. Margam VM, Coates BS, Bayles DO, Hellmich RL, Agunbiade T, Seufferheld MJ, Sun W, Kroemer JA, Ba MN, Binso-Dabire CL, Baoua I, Ishiyaku MF, Covas FG, Srinivasan R, Armstrong J, Murdock LL, Pitttendrigh BR: Transcriptome sequencing, and rapid development and application of SNP markers for the legume pod borer Maruca vitrata (Lepidoptera: Crambidae). PLOS One 2011, 6:e21388.

30. Roberts SB, Hauser L, Seeb LW, Seeb JE: Development of genomic resources for Pacific Herring through targeted transcriptome pyrosequencing. PLOS One 2012, 7:e30908.

31. Savory EA, Adhikari BN, Hamilton JP, Vaillancourt B, Buell CR, Day B: mRNASeq analysis of the Pseudoperonospora cubensis transcriptome during cucumber (Cucumis sativus L.) infection. PLoS One 2012, 7:e35796.

32. Picard pipeline. http://picard.sourceforge.net/.

33. Tariq MA, Kim HJ, Jejelowo O, Pourmand N: Whole-transcriptome RNAseq analysis from minute amount of total RNA. Nucleic Acids Res 2011, 39(18):e120

34. Levin JZ, Yassour M, Adiconis X, Nusbaum C, Thompson DA, Friedman N, Gnirke A, Regev A: Comprehensive comparative analysis of strand-specific RNA sequencing methods. Nat Methods 2010, 7(9):709-715.

35. Kozik A, M M, Kozik I, Van Leeuwen H, Van Deynze A, Michelmore R: Eukaryotic ultra conserved orthologs and estimation of gene capture In EST libraries. Plant and Animal Genomes Conference XVI 2008:6.
36. Duarte JM, Wall PK, Edger PP, Landherr LL, Ma H, Pires JC, Leebens-Mack J, dePamphilis CW: Identification of shared single copy nuclear genes in Arabidopsis, Populus, Vitis and Oryza and their phylogenetic utility across various taxonomic levels. BMC Evol Biol 2010, 10:61.

37. Harismendy O, Ng PC, Strausberg RL, Wang X, Stockwell TB, Beeson KY, Schork NJ, Murray SS, Topol EJ, Levy S, Frazer KA: Evaluation of next generation sequencing platforms for population targeted sequencing studies. Genome Biol 2009, 10:R32.

38. Metzker ML: Sequencing technologies - the next generation. Nat Rev Genet 2010, 11:31-46.

39. Weber AP, Weber KL, Carr K, Wilkerson C, Ohlrogge JB: Sampling the Arabidopsis transcriptome with massively parallel pyrosequencing. Plant Physiol 2007, 144:32-42.

40. Novaes E, Drost DR, Farmerie WG, Pappas GJ Jr, Grattapaglia D, Sederoff RR, Kirst M: High-throughput gene and SNP discovery in Eucalyptus grandis, an uncharacterized genome. BMC Genomics 2008, 9:312.

41. Vega-Arreguin JC, Ibarra-Laclette E, Jimenez-Moraila B, Martinez O, Vielle-Calzada JP, Herrera-Estrella L, Herrera-Estrella A: Deep sampling of the Palomero maize transcriptome by a high throughput strategy of pyrosequencing. BMC Genomics 2009, 10:299.

42. Wall PK, Leebens-Mack J, Chanderbali AS, Barakat A, Wolcott E, Liang H, Landherr L, Tomsho LP, Hu Y, Carlson JE, Ma Hong, Schuster SC, Soltis DE, Soltis PS, Altman N, dePamphilis CW: Comparison of next generation sequencing technologies for transcriptome characterization. BMC Genomics 2009, 10:347.

43. Cantacessi C, Campbell BE, Young ND, Jex AR, Hall RS, Presidente PJA, Zawadzki JL, Zhong W, Aleman-Meza B, Loukas A, Sternberg PW, Gasser RB: Differences in transcription between free-living and $\mathrm{CO} 2$-activated thirdstage larvae of Haemonchus contortus. BMC Genomics 2010, 11:266.

44. Nagalakshmi U, Wang Z, Waern K, Shou C, Raha D, Gerst: The transcriptional landscape of the yeast genome defined by RNA sequencing. Science 2008, 320:1344-1349.

45. Rosenkranz R, Borodina $\mathrm{T}$, Lehrach $\mathrm{H}$, Himmelbauer $\mathrm{H}$ : Characterizing the mouse ES cell transcriptome with Illumina sequencing. Genomics 2008, 92:187-194.

46. Birol I, Jackman SD, Nielsen CB, Qian JQ, Varhol R, Stazyk G, Morin RD, Zhao Y, Hirst M, Schein JE, Horsman DE, Connors JM, Gascoyne RD, Marra MA, Jones SJM: De novo transcriptome assembly with ABySS. Bioinformatics 2009, 25:2872-2877

47. Wang X-W, Luan J-B, Li J-M, Bao Y-Y, Zhang C-X, Liu S-S: De novo characterization of a whitefly transcriptome and analysis of its gene expression during development. BMC Genomics 2010, 11:400.

48. Kumar S, Blaxter ML: Comparing de novo assemblers for 454 transcriptome data. BMC Genomics 2010, 11:571.

49. Feldmeyer B, Wheat CW, Krezdorn N, Rotter B, Pfenninger M: Short read Illumina data for the de novo assembly of a non-model snail species transcriptome (Radix balthica, Basommatophora, Pulmonata), and a comparison of assembler performance. BMC Genomics 2011, 12:317.

50. Brautigam A, Mullick T, Schliesky S, Weber AP: Critical assessment of assembly strategies for non-model species mRNA-Seq data and application of next-generation sequencing to the comparison of $C(3)$ and $C(4)$ species. J Exp Bot 2011, 62:3093-3102.

51. Garg R, Patel RK, Jhanwar S, Priya P, Bhattacharjee A, Yadav G, Bhatia S, Chattopadhyay D, Tyagi AK, Jain M: Gene discovery and tissue-specific transcriptome analysis in chickpea with massively parallel pyrosequencing and web resource development. Plant Physiol 2011, 156:1661-1678.

52. Kaur S, Cogan NO, Pembleton LW, Shinozuka M, Savin KW, Materne M, Forster JW: Transcriptome sequencing of lentil based on secondgeneration technology permits large-scale unigene assembly and SSR marker discovery. BMC Genomics 2011, 12:265.

53. Logacheva MD, Kasianov AS, Vinogradov DV, Samigullin TH, Gelfand MS, Makeev VJ, Penin AA: De novo sequencing and characterization of floral transcriptome in two species of buckwheat (Fagopyrum). BMC Genomics 2011, 12:30.

54. Natarajan P, Parani M: De novo assembly and transcriptome analysis of five major tissues of Jatropha curcas L. using GS FLX titanium platform of 454 pyrosequencing. BMC Genomics 2011, 12:191.

55. Shi CY, Yang H, Wei CL, Yu O, Zhang ZZ, Jiang CJ, Sun J, Li YY, Chen Q, Xia T, et al: Deep sequencing of the Camellia sinensis transcriptome revealed candidate genes for major metabolic pathways of tea-specific compounds. BMC Genomics 2011, 12:131. 
56. Wenping $H$, Yuan $Z$, Jie $S$, Lijun Z, Zhezhi W: De novo transcriptome sequencing in Salvia miltiorrhiza to identify genes involved in the biosynthesis of active ingredients. Genomics 2011, 98:272-279.

57. Schmitz-Linneweber C, Small I: Pentatricopeptide repeat proteins: a socket set for organelle gene expression. Trends Plant Sci 2008, 13:663-670.

58. Chateigner-Boutin AL, Ramos-Vega M, Guevara-Garcia A, Andres C, de la Luz G-NM, Cantero A, Delannoy E, Jimenez LF, Lurin C, Small I, et al: CLB19, a pentatricopeptide repeat protein required for editing of rpoA and $C l p P$ chloroplast transcripts. Plant J 2008, 56:590-602.

59. Cai W, Ji D, Peng L, Guo J, Ma J, Zou M, Lu C, Zhang L: LPA66 is required for editing psbF chloroplast transcripts in Arabidopsis. Plant Physiol 2009, 150:1260-1271.

60. Hammani K, Okuda K, Tanz SK, Chateigner-Boutin AL, Shikanai T, Small I: A study of new Arabidopsis chloroplast RNA editing mutants reveals general features of editing factors and their target sites. Plant Cell 2009, 21:3686-3699.

61. Robbins JC, Heller WP, Hanson MR: A comparative genomics approach identifies a PPR-DYW protein that is essential for C-to- $U$ editing of the Arabidopsis chloroplast accD transcript. RNA 2009, 15:1142-1153.

62. Yu QB, Jiang Y, Chong K, Yang ZN: AtECB2, a pentatricopeptide repeat protein, is required for chloroplast transcript accD RNA editing and early chloroplast biogenesis in Arabidopsis thaliana. Plant J 2009, 59:1011-1023.

63. Zhou W, Cheng Y, Yap A, Chateigner-Boutin AL, Delannoy E, Hammani K, Small I, Huang J: The Arabidopsis gene YS1 encoding a DYW protein is required for editing of $r p o B$ transcripts and the rapid development of chloroplasts during early growth. Plant J 2009, 58:82-96.

64. Tseng CC, Sung TY, Li YC, Hsu SJ, Lin CL, Hsieh MH: Editing of accD and $n d h F$ chloroplast transcripts is partially affected in the Arabidopsis vanilla cream1 mutant. Plant Mol Biol 2010, 73:309-323.

65. Hayes ML, Giang K, Mulligan RM: Molecular evolution of pentatricopeptide repeat genes reveals truncation in species lacking an editing target and structural domains under distinct selective pressures. BMC Evol Biol 2012, 12:66.

66. Lynch M, Koskella B, Schaack S: Mutation pressure and the evolution of organelle genomic architecture. Science 2006, 311(5768):1727-1730.

67. Sloan DB, MacQueen AH, Alverson AJ, Palmer JD, Taylor DR: Extensive loss of RNA editing sites in rapidly evolving Silene mitochondrial genomes: selection vs. retroprocessing as the driving force. Genetics 2010, 185(4):1369-1380.

68. Cuenca A, Petersen G, Seberg O, Davis Jl, Stevenson DW: Are substitution rates and RNA editing correlated? BMC Evol Biol 2010, 10:349.

69. Downie SR, Katz-Downie DS, Wolfe KH, Calie PJ, Palmer JD: Structure and evolution of the largest chloroplast gene (ORF2280): internal plasticity and multiple gene loss during angiosperm evolution. Curr Genet 1994, 25:367-378

70. Palmer JD, Calie PJ, dePamphilis CW, Logsdon JMJ, Katz-Downie DS, Downie SR, Palmer JD, Calie PJ, de Pamphilis CW, Logsdon JMJ, Katz-Downie DS, Downie SR: An evolutionary genetic approach to understanding plastid gene function: lessons from photosynthetic and nonphotosynthetic plants. In Current research in photosynthesis. Edited by Baltscheffsky M, Baltscheffsky M. Amsterdam: Kluwer Academic Publishers; 1990:475-482.

71. Palmer JD, Baldauf SL, Calie PJ, DePamphilis CW: Chloroplast gene instability and transfer to the nucleus. In Molecular evolution. Edited by Clegg MT, O'Brien SJ. New York: Alan R. Liss, Inc; 1990:97-106.

72. Lysenko EA: Plant sigma factors and their role in plastid transcription. Plant Cell Rep 2007, 26:845-859.

73. Meyer E, Aglyamova GV, Wang S, Buchanan-Carter J, Abrego D, Colbourne $J K$, Willis BL, Matz MV: Sequencing and de novo analysis of a coral larval transcriptome using 454 GSFIx. BMC Genomics 2009, 10:219.

74. Lamesch P, Berardini TZ, Li D, Swarbreck D, Wilks C, Sasidharan R, Muller R, Dreher K, Alexander DL, Garcia-Hernandez M, et al: The Arabidopsis Information Resource (TAIR): improved gene annotation and new tools. Nucleic Acids Res 2012, 40(Database issue):D1202-D1210.

75. Langmead B, Salzberg SL: Fast gapped-read alignment with Bowtie 2. Nat Methods 2012, 9(4):357-359.

76. Li H, Handsaker B, Wysoker A, Fennell T, Ruan J, Homer N, Marth G, Abecasis $G$, Durbin R: The sequence alignment/map format and SAMtools. Bioinformatics 2009, 25(16):2078-2079.

77. Grabherr MG, Haas BJ, Yassour M, Levin JZ, Thompson DA, Amit I, Adiconis X, Fan L, Raychowdhury R, Zeng Q, Chen Z, Mauceli E, Hacohen N, Gnirke A, Rhind N, di PLM F, Birren BW, Nusbaum C, Lindblad-Toh K, Friedman N, Regev A: Full-length transcriptome assembly from RNA-Seq data without a reference genome. Nat Biotech 2011, 29:644-652.
78. Li R, Li Y, Kristiansen K, Wang J: SOAP: short oligonucleotide alignment program. Bioinformatics 2008, 24:713-714.

79. Li R, Zhu H, Ruan J, Qian W, Fang X, Shi Z, Li Y, Li S, Shan G, Kristiansen K, Li S, Yang H, Wang J, Wang J: De novo assembly of human genomes with massively parallel short read sequencing. Genome Res 2010, 20:265-272.

80. Chevreux B, Pfisterer T, Drescher B, Driesel AJ, Muller WEG, Wetter T, Suhai S: Using the miraEST assembler for reliable and automated mRNA transcript assembly and SNP detection in sequenced ESTs. Genome Res 2004, 14:1147-1159.

81. Margulies M, Egholm M, Altman WE, Attiya S, Bader JS, Bemben LA, Berka J, Braverman MS, Chen YJ, Chen Z, Dewell SB, Du L, Fierro JM, Gomes XV, Godwin BC, He W, Helgesen S, Ho CH, Irzyk GP, Jando SC, Alenquer MLI, Jarvie TP, Jirage KB, Mim J-B, Knight JR, Lanza JR, Leamon JH, Lefkowitz SM, et al: Genome sequencing in microfabricated high-density picolitre reactors. Nature 2005, 437:376380.

82. Li W, Godzik A: CD-HIT: a fast program for clustering and comparing large sets of protein or nucleotide sequences. Bioinformatics 2006, 22:1658-1659.

83. Camacho C, Coulouris G, Avagyan V, Ma N, Papadopoulos J, Bealer K, Madden TL: BLAST+: architecture and applications. BMC Bioinforma 2009, 10:421.

84. Edgar RC: MUSCLE: multiple sequence alignment with high accuracy and high throughput. Nucleic Acids Res 2004, 32:1792-1797.

85. Martin JA, Wang Z: Next-generation transcriptome assembly. Nat Rev Genet 2011, 12:671-682.

86. Boeckmann B, Bairoch A, Apweiler R, Blatter MC, Estreicher A, Gasteiger E, Martin MJ, Michoud K, O'Donovan C, Phan I, Pilbout S, Schneider M: The SWISS-PROT protein knowledgebase and its supplement TrEMBL in 2003. Nucleic Acids Res 2003, 31:365-370.

87. Conesa A, Gotz S: Blast2GO: a comprehensive suite for functional analysis in plant genomics. Int J Plant Genomics 2008, 2008:619832

88. Conesa A, Gotz S, Garcia-Gomez JM, Terol J, Talon M, Robles M: Blast2GO: a universal tool for annotation, visualization and analysis in functional genomics research. Bioinformatics 2005, 21:3674-3676.

89. Gotz S, Garcia-Gomez JM, Terol J, Williams TD, Nagaraj SH, Nueda MJ, Robles M, Talon M, Dopazo J, Conesa A: High-throughput functional annotation and data mining with the Blast2GO suite. Nucleic Acids Res 2008 36:3420-3435

90. Eddy SR: Profile hidden Markov models. Bioinformatics 1998, 14:755-763.

91. Lurin C, Andres C, Aubourg S, Bellaoui M, Bitton F, Bruyere C, Caboche M, Debast C, Gualberto J, Hoffmann B, Lecharnt A, Ret ML, Martin-Magniette M-L, Mireau H, Peeters N, Renou J-P, Szurek B, Taconnat L, Small I: Genomewide analysis of Arabidopsis pentatricopeptide repeat proteins reveals their essential role in organelle biogenesis. Plant Cell 2004, 16:2089-2103.

92. Small ID, Peeters N: The PPR motif - a TPR-related motif prevalent in plant organellar proteins. Trends Biochem Sci 2000, 25:46-47.

93. Helmann JD, Chamberlin MJ: Structure and function of bacterial sigma factors. Annu Rev Biochem 1988, 57:839-872.

94. Isono K, Shimizu M, Yoshimoto K, Niwa Y, Satoh K, Yokota A, Kobayashi H: Leaf-specifically expressed genes for polypeptides destined for chloroplasts with domains of sigma70 factors of bacterial RNA polymerases in Arabidopsis thaliana. Proc Natl Acad Sci U S A 1997, 94:14948-14953.

95. Hakimi MA, Privat I, Valay J-G, Lerbs-Mache S: Evolutionary conservation of C-terminal domains of primary sigma(70)-type transcription factors between plants and bacteria. J Biol Chem 2000, 275:9215-9221.

96. Nielsen H, Engelbrecht J, Brunak S, Heline G: Identification of prokaryotic and eukaryotic signal peptides and prediction of their cleavage sites. Protein Eng 1997, 10:1-6.

97. Emanuelsson O, Nielsen H, Brunak S, Heline G: Predicting subcellular localization of proteins based on their $\mathrm{N}$-terminal amino acid sequence. $\mathrm{J} \mathrm{Mol} \mathrm{Biol} \mathrm{2000,} \mathrm{300:1005-1016.}$

doi:10.1186/1471-2229-13-228

Cite this article as: Zhang et al: Comparative analyses of two Geraniaceae transcriptomes using next-generation sequencing. BMC Plant Biology 2013 13:228. 